\title{
Urinary Neutrophil Gelatinase-Associated Lipocalin Is a Promising Biomarker for Late Onset Culture-Positive Sepsis in Very Low Birth Weight Infants
}

\author{
ELVIRA PARRAVICINI, SHERI L. NEMEROFSKY, KENNETH A. MICHELSON, TRANG K. HUYNH, MEGHAN E. SISE, \\ DAVID A. BATEMAN, JOHN M. LORENZ, AND JONATHAN M. BARASCH
}

\begin{abstract}
Department of Pediatrics [E.P., D.A.B., J.M.L.], Department of Medicine [K.A.M., M.E.S., J.M.B.], College of Physicians and Surgeons, Columbia University, New York, New York 10032; Department of Pediatrics [S.L.N.], Albert Einstein College of Medicine/Montefiore Medical Center, Bronx, New York 10461; Department of Pediatrics [T.K.H.], New York Presbyterian-Weill Cornell Medical College, New York,
\end{abstract} New York 10065.

\begin{abstract}
Need for the early identification of sepsis in very low birth weight (VLBW) infants has led to the search for reliable biomarkers. This study aims to determine whether urinary neutrophil gelatinase-associated lipocalin (UNGAL) rises in culture-positive sepsis and, if so, is elevated at the time sepsis is suspected. This is a prospective study of 91 VLBW infants whose urine was collected daily for uNGAL analysis. In 65 episodes of suspected sepsis, four groups were identified: a) culture-positive sepsis; b) single culture positive for Staphylococcus epidermidis; c) and d) negative culture with antibiotic treatment for $\geq 7 \mathrm{~d}$ and $<7 \mathrm{~d}$, respectively. Daily means of uNGAL of each group were estimated for comparison. Mean uNGAL in group A (179 $\mathrm{ng} / \mathrm{mL})$ was significantly elevated on the day blood culture was drawn (day 0) compared with the mean of healthy VLBW infants $(6.5 \mathrm{ng} / \mathrm{mL})$, and to the means in groups $\mathrm{B}, \mathrm{C}$, and $\mathrm{D}(p<0.05)$. In group $\mathrm{A}$, mean uNGAL was significantly elevated on day 0 and daily for 5 days when compared with that of the day before culture $(p<0.05$ to $<0.005)$. uNGAL shows promise as an early marker for culture-positive sepsis in VLBW infants. (Pediatr Res 67: 636-640, 2010)
\end{abstract}

$\mathrm{V}$ ery low birth weight (VLBW) infants are at substantial risk of late onset (occurring after day of life four) sepsis (1-4). The incidence of sepsis has recently been reported to be as high as $42 \%$ with a mortality rate of $18 \%$ in some NICU subpopulations (5). The diagnosis of sepsis in premature infants is notoriously difficult because of its nonspecific signs and symptoms in this population, the difficulties obtaining adequate samples for blood culture, and frequent co-morbid conditions that can mask, mimic, or accompany sepsis. As a result, empiric antibiotic therapy for late onset sepsis is frequently prescribed $(6,7)$ with the potential for concomitant toxicity and the emergence of resistance (8).

Several early biomarkers for sepsis in the neonatal population have been proposed. Quantitative C-reactive protein (CRP) measurement is widely used in sepsis screening in newborn infants. CRP sensitivity is low at the time of first

Received November 16, 2009; accepted February 1, 2010.

Correspondence: Elvira Parravicini, MD, Department of Pediatrics, 3959 Broadway, New York, NY 10032; e-mail: ep127@columbia.edu

Supported by Grant Number UL1 RR024156 and grants from the Emerald Foundation, the March of Dimes and the NIDDK (Grants DK-55388 and DK-58872) to J.M.B.

S.L.N. and K.A.M. contributed equally to this study. clinical suspicion but improves with two repeated measurements at 12-24 h intervals. However, its positive predictive value remains low (9), and it is less reliable in preterm infants (10). Many other cytokines or acute phase reactants have also been studied, but similarly lack sensitivity unless measured serially or in combination with other tests (11).

An ideal biomarker for infection in newborns should be quantifiable using a small, easily obtained body fluid sample; be elevated early in the course of infection; and have an adequate window of opportunity for sampling, i.e., a sufficient period during which its level remains abnormal. In addition, the marker should be biochemically stable and have minimal transportation requirements. The analytical procedure should have a short turnaround time, low cost, and be available around the clock. The results should be comparable among different laboratories. Most importantly, a clinically useful infection marker must have a well defined threshold value for differentiating infected from noninfected infants and be able to identify infected infants at an early stage. A very high sensitivity and negative predictive value (approaching 100\%) and good specificity and positive predictive value $(>85 \%)$ are desirable. The ability to differentiate various types of infection (bacterial, fungal, or viral) would be advantageous. Further, it should be able to reflect the progress of the disease and guide the use of antimicrobial therapy.

Neutrophil gelatinase-associated lipocalin (NGAL) is a 25$\mathrm{kDa}$ protein expressed at very low levels in neutrophils and several other human tissues, including the lung, gastrointestinal tract, and kidney. Expression of this protein rises dramatically when epithelial organs undergo signaling, which is usually associated with cell damage, including ischemiareperfusion injury (12), the presence of cytotoxins (12), and sepsis (13). In the setting of these insults, NGAL is upregulated in the circulation and in the urine. The source of serum NGAL includes neutrophils, the liver, and the spleen; the source of urinary NGAL is predominately the kidney tubule $(13,14)$. In the normal kidney circulating NGAL is

\footnotetext{
Abbreviations: AKI, acute kidney injury; CRP, C-reactive protein; NGAL, neutrophil gelatinase-associated lipocalin; UNGAL, urinary neutrophil gelatinase-associated lipocalin; VLBW, very low birth weight
} 
filtered by the glomerulus but is then degraded in the proximal tubule $\left(T_{1 / 2}=10 \mathrm{~min}\right)$ after capture by the megalin complex, as demonstrated in knockout mice; only a small amount $(0.1-0.2 \%)$ is excreted in urine (15). In contrast, urinary NGAL (uNGAL) derives mostly from expression of NGAL message in thick limbs of Henle and collecting ducts in both the postischemic and postseptic kidney $(13,14)$. In the setting of these diseases in mouse models, NGAL message increases over 1000 fold and NGAL protein is readily obtained from urine specimens.

Clinical studies in children and adults have shown that in setting of bacterial infection serum NGAL is markedly increased (16). Patients with acute kidney injury (AKI) associated with bacterial infection have the highest levels of serum and urinary NGAL $(15,17)$. Although the mechanism of expression of UNGAL in ischemic disease is not understood, the expression of UNGAL in response to both Gram-positive and Gram-negative components is dependent on Toll-like receptor (TLR)-NF-kB signaling, which produces a rapid transcriptional response (18). Hence, because the diagnosis of sepsis in neonates is nearly impossible to confirm at the time when clinical suspicion first arises, we determined whether uNGAL increases in response to systemic sepsis in VLBW infants and whether it might be useful as an early biomarker of disease.

\section{METHODS}

Study subjects. This is a prospective, observational study of VLBW infants (birth weight $<1500 \mathrm{~g}$ ) inborn at Morgan Stanley Children's Hospital of New York-Presbyterian (MSCHONY), Columbia University Medical Center from March 2007 to March 2008 and Children's Hospital at Montefiore (CHAM), Albert Einstein College of Medicine from November 2007 to March 2008. This cohort is a subsample of 145 inborn adequate for gestational age (AGA) premature VLBW newborns. Because one purpose of the larger observational study was to define a reference range for uNGAL in VLBW infants with uncomplicated courses, as previously reported $(19,20)$, small for gestational age (SGA) infants were prospectively excluded; other exclusion criteria were major congenital anomalies, abnormal karyotype, or anticipation of early death or transfer to a referring institution. Parents were approached to participate in the study during their infant's first week of life during work hours from Monday to Friday. The institutional review boards of MSCHONY and CHAM approved the study with documentation of informed consent from participants' parents.

NGAL assay. Bagged spot urine specimens ( $\geq 0.5 \mathrm{~mL}$ ) were collected on the day of enrollment and daily until 32-wk postmenstrual age, discharge, or death-which ever occurred first. The assay used has been previously described (20). In brief, urine was centrifuged (5000 rpm, $5 \mathrm{~min}$ ) and supernatant stored at $-80^{\circ} \mathrm{C}$. NGAL concentration was determined by immunoblot. Because this is a pilot study based on the measurement of NGAL in a population of patients that has not been previously quantified, we chose a rigorous method of identifying and quantifying the canonical NGAL protein (23-25 KDa). Immunoblot is used to certify the measurement of the authentic NGAL monomer and to distinguish it from other forms of NGAL, which are measured along with the monomer in some published studies of urine NGAL (21). The immunoblot technique has been verified by the use of multiple antibodies, the isolation and sequencing of the monomeric protein, and additionally by a comparison with a standardized commercial platform (Abbott), which detects authentic monomeric NGAL. Urinary NGAL analyses were performed in batches days to weeks after collection and medical caregivers were unaware of the results.

Case definitions and data collection. Late onset culture positive sepsis was defined as presence of one or more blood cultures positive for a pathogen or two or more blood cultures positive for Staphylococcus epidermidis after day of life four. Episodes of late onset culture positive sepsis were ascertained by daily review of the clinical course of each infant, together with review of their electronic medical records. In addition to episodes of culture proven sepsis (group A), three additional categories of suspected sepsis were identified for comparison: a) episodes of single positive blood cultures for $S$. epidermidis; b) episodes with negative blood cultures and $\geq 7 \mathrm{~d}$ of antibiotic therapy; and c) episodes with negative blood cultures with $<7 \mathrm{~d}$ of antibiotic therapy. Episodes of sepsis-related AKI, defined as an increase in serum creatinine $\geq 0.3 \mathrm{mg} / \mathrm{dL}$ sustained for at least $48 \mathrm{~h}$ during treatment for culture positive sepsis, were also identified.

Serum creatinine was measured in the institutions' clinical laboratories by the Jaffe kinetic reaction as per standard methodology. Sepsis evaluations were undertaken at the discretion of the attending medical team.

Reference ranges for uNGAL concentrations in VLBW infants were determined from urine samples from infants with uncomplicated clinical courses as previously reported (20). These infants were recruited, enrolled, and had their urine collected and analyzed concurrently with study infants who were evaluated for sepsis.

Statistical analysis. Mean UNGAL concentrations of group A on day 0 and day -1 were compared with the values of healthy VLBW infants previously reported (20) and with the values of the other three groups of episodes of suspected sepsis (B, C, and D) using linear models adjusted for birth weight and gender. Linear mixed models with random intercepts for subjects, where the outcome modeled was $\log (\mathrm{uNGAL})$, were used to estimate the mean and 95\% CI for $\log (\mathrm{uNGAL})$ on each day from $5 \mathrm{~d}$ before to $5 \mathrm{~d}$ after the day that blood cultures were obtained (day 0) in infants with culture proven sepsis (group A); results were exponentiated back to absolute scale. Therefore, "mean uNGAL" values reported from regression models as well as throughout are geometric means. Results from these models were used to delineate the pattern of mean uNGAL over the time interval $\pm 5 \mathrm{~d}$ from the day of blood cultures, using the value on day 1 as reference.

Receiver-operator curves (ROC) were constructed to evaluate the ability of uNGAL to discriminate between episodes of confirmed sepsis (group A) and episodes with negative blood culture and treatment $<7 \mathrm{~d}$ (group D) on DOL 0, for sexes combined (shown in Fig. 3) and for males and females separately using SPSS software (SPSS Inc, Chicago IL 60606, www.spss.com). Areas under the curve (AUC) values and their 95\% CIs were determined for sexes combined and separately. To estimate the sensitivity and specificity of a hypothetical screening test based on current data, uNGAL values at the 99 percentile of the reference ranges for males and females (20) were combined into a single indicator variable and cross-tabulated against the results of confirmed positive and confirmed negative blood cultures (groups A and D).

\section{RESULTS}

Ninety-one VLBW infants were recruited for this study within the first $7 \mathrm{~d}$ of life; 61 were enrolled at MSCHONY and 30 at CHAM. During the study periods, 122 inborn infantsVLBW infants were not included in the study for the following reasons: the parents of 42 were not approached because a study team member or parents were unavailable, the parents of 29 declined to participate, 23 were small for gestational age, 14 died on the first day of life, 5 had congenital anomalies, and transfer to a referring institution of 8 was anticipated. In addition, one infant's parents withdrew consent.

Clinical characteristics of infants in the four sepsis groups are shown in Table 1 . In nearly all cases, vancomycin and gentamicin were initially administered and then antibiotic therapy was adjusted as appropriate based on culture results. Sixteen infants (group A) had 16 episodes of culture-proven sepsis: eight due to $S$. aureus, two due to $S$. epidermidis, three due to Gram-negative bacilli, and three due to Candida species. Of these 16, three had associated AKI; none had necrotizing enterocolitis.

Geometric means and $95 \%$ CI of uNGAL concentrations $(\mathrm{ng} / \mathrm{mL}$ ) for the four categories of suspected late-onset sepsis on day 0 were as follows: group A (culture-positive sepsis): 179 (100,318); group B (single positive blood culture for $S$. epidermidis): 62 (24,160); group C (negative blood cultures with $\geq 7 \mathrm{~d}$ of antibiotic therapy): 37 (17,79); and group D (negative culture with $<7 \mathrm{~d}$ of antibiotic therapy): $22(14,35)$. These are displayed in Fig. 1, along with the upper bound of 
Table 1. Clinical characteristics of study infants according to suspected sepsis group and infants with uncomplicated clinical course previously reported (20)

\begin{tabular}{|c|c|c|c|c|c|}
\hline & $\begin{array}{l}\text { Uncomplicated clinical } \\
\text { course }\end{array}$ & $\begin{array}{l}\text { A: Culture positive } \\
\text { sepsis }\end{array}$ & $\begin{array}{l}\text { B: Single positive culture } \\
\text { for } S \text {. epidermidis }\end{array}$ & $\begin{array}{l}\text { C: Culture negative, } \\
\text { treated } \geq 7 \text { days }\end{array}$ & $\begin{array}{c}\text { D: Culture negative, } \\
\text { treated }<7 \text { days }\end{array}$ \\
\hline Number of babies & 50 & 16 & 8 & 4 & 37 \\
\hline $\mathrm{M}: \mathrm{F}$ & 1.5 & 0.8 & 0.6 & 1 & 2.6 \\
\hline Gestational age (wk)* & $28.8 \pm 2.3$ & $25.9 \pm 1.9 \dagger$ & $26.4 \pm 1.9 \dagger$ & $27.0 \pm 4.3$ & $26.0 \pm 1.3 \dagger$ \\
\hline Birth weight $(\mathrm{g})^{*}$ & $1145 \pm 259$ & $875 \pm 254 \dagger$ & $824 \pm 249$ & $981 \pm 409 \dagger$ & $900 \pm 197 \dagger$ \\
\hline Day of life of blood culture $\ddagger$ & - & $19(8-72)$ & $19(10-48)$ & $13(6-20)$ & $21(5-62)$ \\
\hline
\end{tabular}

*Mean \pm standard deviation.

$\dagger p<0.05$ compared to infants with uncomplicated clinical courses as determined by post-hoc testing (Dunnet method).

$\ddagger$ Median (range).

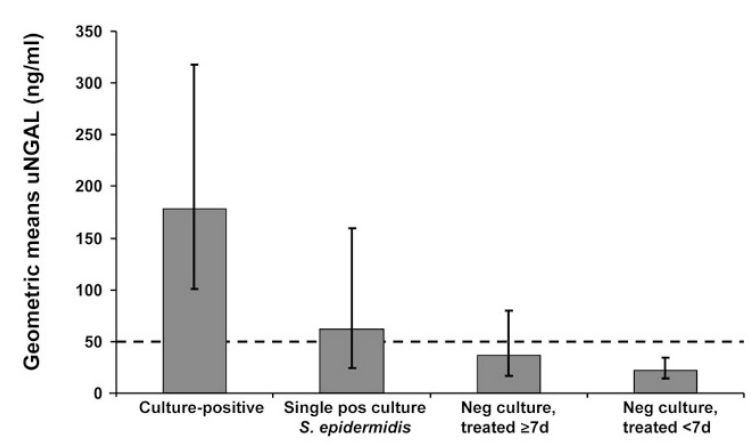

Figure 1. Geometric mean uNGAL concentrations and 95\% CIs on day blood culture drawn in VLBW infants for four culture-outcome groups A-D. The dashed line represents the upper bound of the 95\% CI of uNGAL (55 $\mu \mathrm{g} / \mathrm{mL}$ ) from VLBW infants with uncomplicated clinical courses.

the $95 \%$ confidence limit of the reference range $(55 \mathrm{ng} / \mathrm{mL})$. Mean uNGAL concentration was significantly higher in the culture positive sepsis group (group A) than in the group with negative cultures and treatment $<7 \mathrm{~d}$ (group D). CIs for groups $\mathrm{A}$ and $\mathrm{D}$, respectively, lay well above and below the upper bound of the $95 \% \mathrm{CI}$ of the reference range. A linear regression model controlling for birth weight and gender demonstrated significant differences between values of mean $\log$ (uNGAL) in group A and each of the other three groups. On day 1, the mean UNGAL value of group A was lower than on day 0 [57 (31-106) versus 179 (100-318), for mean and $95 \% \mathrm{CI}$ in $\mathrm{ng} / \mathrm{mL}$, respectively], but still differed significantly from the value of group D on day 1 in the linear regression model controlling for birth weight and gender. Of note, the three infants with late-onset culture positive sepsis, who developed concurrent AKI, had UNGAL concentrations in the range $100-1500 \mathrm{ng} / \mathrm{mL}$ on day 0 .

For infants in group A, the pattern of UNGAL concentration over time relative to the day when blood cultures were obtained (day 0) is shown in Fig. 2. Mean uNGAL concentrations on days 5 to 1 were similar. The uNGAL concentration on day 0 was significantly greater than on day 1 , as were the concentrations on day +1 to $+5(n=16 ; p<0.05$ to $<0.005)$. In contrast, in group D infants, mean uNGAL on day 0 was not elevated compared with the reference range, nor to the day 1 level $(n=37 ; p=0.60)$.

ROCs were constructed to evaluate the ability of UNGAL to discriminate between episodes of confirmed sepsis (group A) and episodes with negative blood culture and treatment $<7 \mathrm{~d}$

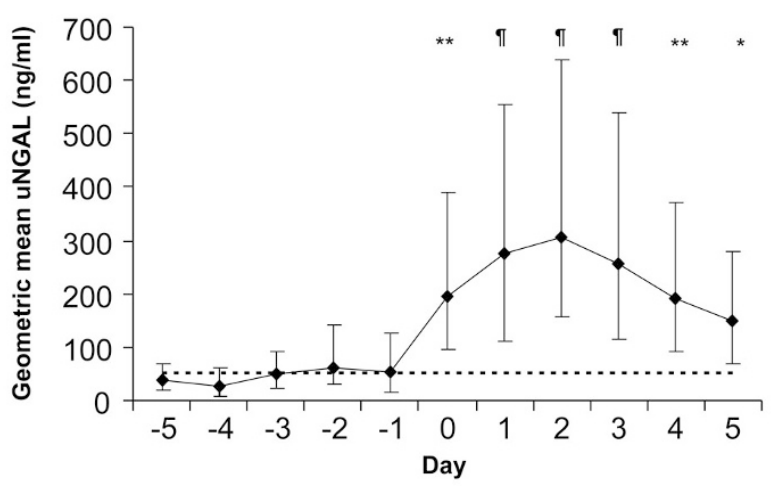

Figure 2. Daily geometric mean uNGAL concentrations and $95 \%$ CIs for VLBW infants with culture-positive sepsis from $5 \mathrm{~d}$ before $5 \mathrm{~d}$ after the blood culture were drawn. The dashed line represents the upper bound of the $95 \%$ CI of uNGAL from VLBW infants with uncomplicated clinical courses. Significant differences of each day from day 1 are denoted as $* p<0.05$, $* * p<0.005, \mathbb{I} p<0.0005$.

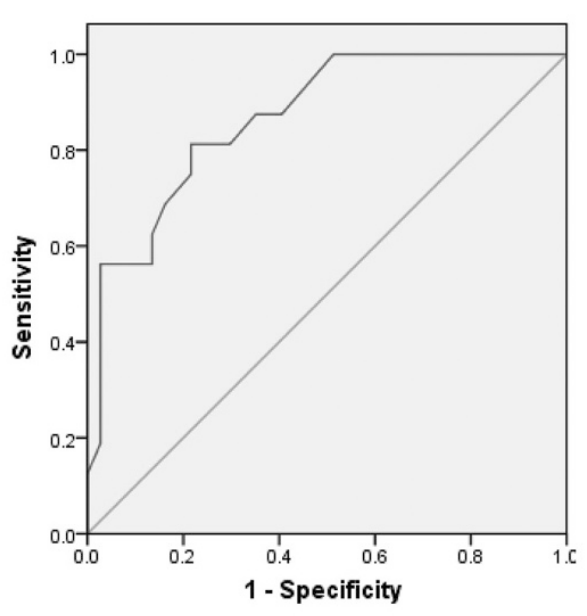

Figure 3. ROC for uNGAL on day blood culture drawn showing the ability of uNGAL to discriminate between episodes of culture proven sepsis (group A) and episodes of negative blood culture and treatment $<7$ d (group D) for both males and females. AUC and $95 \% \mathrm{CI}$ for sex combined are 0.87 (0.77-0.97).

(group D) on DOL 0, for sexes combined (shown in Fig. 3) and for males and females separately. AUC values and their 95\% CIs for sexes combined, for males, and for females are 0.87 (0.77-0.97), $0.87(0.69-1.0)$, and 0.83 (0.65-1.0), respectively. When uNGAL values of $75 \mathrm{ng} / \mathrm{mL}$ for males $(<99$ th percentile of male reference range $)$ and $130 \mathrm{ng} / \mathrm{mL}$ for 
females are used ( $~ 99$ th percentile of female reference range) (20) and combined into a single gender dependent indicator variable, the resulting test has the following characteristics: odds ratio, $15.5(3.71-65) ; \chi^{2}, 17.2(p<0.0001)$; sensitivity, $12 / 16=$ 0.75 (0.51-0.89); specificity, $31 / 37=0.84(0.69-0.92)$; positive predictive value (PPV), 12/18 $=0.67(0.44-0.84)$; negative predictive value (NPV), 31/35 $=0.89(0.74-0.95)$.

\section{DISCUSSION}

To our knowledge, this is the first peer-reviewed prospective study to investigate the role of uNGAL as a biomarker for culture-positive sepsis in VLBW infants. Infants with culture proven sepsis expressed UNGAL at levels approximately 30fold higher than healthy infants. Moreover, uNGAL was elevated on the same day that blood cultures were obtained. That uNGAL concentration was not elevated in patients whose positive cultures were presumed to be due to contamination or were negative, suggests that the uNGAL assay may have a useful negative predictive value for sepsis. The time course of elevation of uNGAL in relation to the day the blood cultures were obtained demonstrates the rapid expression of uNGAL at the time sepsis was first suspected clinically and its persistence and decline thereafter with treatment.

According to clinical studies in adults and children $(15,16)$, serum NGAL is elevated with sepsis, but our results suggest that the kidney also responds to sepsis by up-regulating uNGAL production. It is unlikely that much of this protein found in the urine derives from circulating NGAL filtered by the glomerulus, given that in animal model serum NGAL is cleared by the proximal tubule (13). Although it is known that tubule expression of NGAL is up-regulated by diseases that also reduce the GFR, in our study only three out of 16 infants had a concomitant episode of reduced GFR as defined by increased and sustained plasma creatinine by $\leq 0.3 \mathrm{mg} / \mathrm{dL}$. This suggests that, when sepsis was first suspected, uNGAL was up-regulated directly by bacterial products themselves or by circulating inflammatory mediators acting on the distal tubule where NGAL is synthesized $(13,14)$, even in the absence of an effect of these agents on glomerular function. NGAL is known to be selectively up-regulated by IL-1ß and by agonists of TLR TLR4 and TLR2 in epithelial cells (22). Hence, the expression of uNGAL in the setting of sepsis without loss of GFR is consistent with its known molecular signal transduction. Indeed, because of the robust biologic response of NGAL to IL-1 $\beta$ and TLR agonists, a great increase in concentration of uNGAL is expected in infants with culture-positive sepsis.

uNGAL is best known for its association with ischemia, cytotoxicity, and sepsis. In a study of more than 650 adults presenting to an inner city Emergency Department, sepsis produced the highest levels of UNGAL especially when it was associated with a reduced GFR (23). Indeed, the UNGAL concentration in three of our patients with culture positive sepsis associated with reduced GFR was as much as eight times higher than that of the other 13 infants with culture positive sepsis, who had no associated increase in serum creatinine. However, the fact that the rise in uNGAL generally occurred without meeting our criteria for reduced GFR, confirms the direct effect of sepsis on the renal tubule.

Zappitelli et al. (17) studied a heterogeneous pediatric population (age 1 mo to $21 \mathrm{y}$ ) and found no difference in uNGAL concentration in septic patients with or without positive blood culture. UNGAL concentration was elevated in septic patients only in presence of AKI. However, their definition of sepsis included patients diagnosed with sepsis either on admission or discharge. This is a very broad definition including children with proven sepsis and children in whom sepsis was clinically suspected or was ruled out during the admission. In contrast, the focus of our study was testing the ability of uNGAL to identify infants with episodes of culture proven sepsis.

In the newborn population uNGAL has several properties that potentially may complement or improve on those of other available biomarkers of newborn sepsis. A study in a pediatric population with bacterial infection comparing the kinetics of CRP, a widely used biomarker of acute inflammation and serum NGAL found a concomitant rise of both the markers with bacterial infection, however, NGAL was noted to be a better marker for monitoring antibiotic treatment, because its serum levels were more rapidly reversible than CRP during the course of treatment (16). In our data, the rapid decrement of uNGAL levels after treatment is also evident (Fig. 2). The data also suggests that, at least in some cases, uNGAL is expressed earlier in true sepsis than the signs of disease are evident in the infant. Indeed, the mean uNGAL value on day 1 in group A infants lies above the upper bound of normal and significantly exceeds the mean day 1 value of infants whose cultures proved negative (group D). The time scale of our study is measured in days; however, septic premature newborns may deteriorate over hours, sometimes minutes. A more precise timing of the collection of the UNGAL specimen in relation to the presentation of disease signs and the timing of the blood cultures would be of great interest in defining the sensitivity of uNGAL in the early identification of sepsis in VLBW babies.

In the newborn CRP is sometimes used as a negative predictor of early-onset sepsis; in other words, a nonelevated CRP result bolsters the case for stopping treatment in a newborn whose blood culture is negative and whose signs and symptoms may be ambiguous. The role of CRP in identifying nosocomial sepsis (occurring after $72 \mathrm{~h}$ age) is not welldefined. Our study focused on suspected sepsis arising after $4 \mathrm{~d}$ of age. Thus, our data suggests that uNGAL may fill the needed role as a marker for late-onset disease. Thus, our study focused on suspected sepsis arising after $4 \mathrm{~d}$ of age; all the cases of proven sepsis met the definition of late-onset disease. Another potential advantage is that UNGAL can be detected in a very small amount of urine collected under noninvasive and nonsterile conditions and, in contrast to CRP, requires no blood specimen. This property of UNGAL is important for tiny premature infants in whom vascular access is difficult and whose limited blood volume can be easily depleted by specimen drawing.

Based on these preliminary data, when uNGAL values of 75 $\mathrm{ng} / \mathrm{mL}$ for males and $130 \mathrm{ng} / \mathrm{mL}$ for females are combined 
into a single indicator variable, the sensitivity, specificity, and NPV are relatively high. These preliminary estimates for uNGAL are certainly comparable, if not superior, to those of the first CRP taken at the onset of late-onset sepsis (9). However, these results are based on a relatively small number of infants with sepsis and should be regarded as exploratory. Of course, it is possible to generate a different balance of sensitivity and specificity by altering our cutoff values depending on the clinical circumstances in which the test may be used.

One limitation of our study relates to the absence of a strict, agreed-upon definition of loss of GFR in neonates that takes into account the dynamic and gestational-age dependent nature of creatinine concentration in newborns. As the modified criteria for pediatric population of risk, injury, failure, loss, and end-stage renal disease (24) have not yet been proven in VLBW infants population, our definition of AKI (a sustained rise in serum creatinine of $0.3 \mathrm{mg} / \mathrm{dL}$ ) must be considered exploratory. Hence, this cut-off may be overly exclusive, leading us to mistakenly categorize subjects with mild forms of glomerular compromise as having normal function. Although this may have no impact on the utility of uNGAL as a biomarker for sepsis, it may hamper our understanding of the clinical situations in which UNGAL is expressed.

Finally, we envision a potential application of the use of uNGAL that can be critical in preventing morbidity and mortality in this fragile population. Because there is no gold standard for diagnosing sepsis the day it occurs (as opposed to when positive cultures are reported) and UNGAL may detect sepsis better than clinical judgment, we propose to measure uNGAL as soon as the first nonspecific symptoms appear.

In conclusion, uNGAL is a promising candidate for an early biomarker of sepsis in VLBW infants. However, a larger prospective study is required to determine the optimal UNGAL threshold value that maximizes its ability to identify true sepsis (or true nonsepsis) in infants with clinical signs consistent with disease, as is more information about what other conditions might elevate UNGAL concentration in VLBW infants.

Acknowledgments. We thank Nicholas Barasch, Theresa Bowman, Charles Buchen, Kristin M. Capone, Catherine Forster, Shlomo Kuperman, Stephanie Marion, Elena Polesana, Allison Polland, Patrick L. Scarborough, and Peter Stella, for their assistance in obtaining daily urine samples, NGAL assay, and data collections; and Jimmy Duong, MPH from the Columbia University Center for Clinical and Translational Research for his contributions to the data analysis.

\section{REFERENCES}

1. Stoll BJ, Hansen N, Fanaroff AA, Wright LL, Carlo WA, Ehrenkranz RA, Lemons JA, Donovan EF, Stark AR, Tyson JE, Oh W, Bauer CR, Korones SB, Shankaran S, Laptook AR, Stevenson DK, Papile LA, Poole WK 2002 Late-onset sepsis in very low birth weight neonates: the experience of the NICHD Neonatal Research Network. Pediatrics 110:285-291

2. Bizzarro MJ, Raskind C, Baltimore RS, Gallagher PG 2005 Seventy-five years of neonatal sepsis at Yale:1928-2003. Pediatrics 116:595-602

3. Graham PL III, Begg MD, Larson E, Della-Latta P, Allen A, Saiman L 2006 Risk factors for late onset gram-negative sepsis in low birth weight infants hospitalized in the neonatal intensive care unit. Pediatr Infect Dis J 25:113-117

4. Fanaroff AA, Stoll BJ, Wright LL, Carlo WA, Ehrenkranz RA, Stark AR, Bauer CR, Donovan EF, Korones SB, Laptook AR, Lemons JA, Oh W, Papile LA, Shankaran S, Stevenson DK, Tyson JE, Poole WK; NICHD Neonatal Research Network 2007 Trends in neonatal morbidity and mortality for very low birthweight infants. Am J Obstet Gynecol 196:147.e1-e8

5. Brodie SB, Sands KE, Gray JE, Parker RA, Goldmann DA, Davis RB, Richardson DK 2000 Occurrence of nosocomial bloodstream infections in six neonatal intensive care units. Pediatr Infect Dis J 19:56-65

6. Apisarnthanarak A, Holzmann-Pazgal G, Hamvas A, Olsen MA, Fraser VJ 2004 Antimicrobial use and the influence of inadequate empiric antimicrobial therapy on the outcomes of nosocomial bloodstream infections in a neonatal intensive care unit. Infect Control Hosp Epidemiol 25:735-741

7. Rubin LG, Sánchez PJ, Siegel J, Levine G, Saiman L, Jarvis WR; Pediatric Prevention Network 2002 Evaluation and treatment of neonates with suspected late-onset sepsis: a survey of neonatologists' practices. Pediatrics 110:e42

8. Calil R, Marba ST, von Nowakonski A, Tresoldi AT 2001 Reduction in colonization and nosocomial infection by multiresistant bacteria in a neonatal unit after institution of educational measures and restriction in the use of cephalosporins. Am J Infect Control 29:133-138

9. Benitz WE, Han MY, Madan A, Ramachandra P 1998 Serial serum C-reactive protein levels in the diagnosis of neonatal infection. Pediatrics 102:E41

10. Hengst JM 2003 The role of C-reactive protein in the evaluation and management of infants with suspected sepsis. Adv Neonatal Care 3:3-13

11. Ng PC, Lam HS 2006 Diagnostic markers for neonatal sepsis. Curr Opin Pediatr 18:125-131

12. Mishra J, Ma Q, Prada A, Mitsnefes M, Zahedi K, Yang J, Barasch J, Devarajan P 2003 Identification of neutrophil gelatinase-associated lipocalin as a novel early urinary biomarker for ischemic renal injury. J Am Soc Nephrol 14:2534-2543

13. Schmidt-Ott KM, Mori K, Kalandadze A, Li JY, Paragas N, Nicholas T, Devarajan $\mathrm{P}$, Barasch J 2006 Neutrophil gelatinase-associated lipocalin-mediated iron traffic in kidney epithelia. Curr Opin Nephrol Hypertens 15:442-449

14. Schmidt-Ott KM, Mori K, Li JY, Kalandadze A, Cohen DJ, Devarajan P, Barasch J 2007 Dual action of neutrophil gelatinase-associated lipocalin. J Am Soc Nephrol 18:407-413

15. Mori K, Lee HT, Rapoport D, Drexler I, Foster K, Yang J, Schmidt-Ott KM, Chen X, Li JY, Weiss S, Mishra J, Cheema FH, Markowitz G, Suganami T, Sawai K, Mukoyama M, Kunis C, D'Agati V, Devarajan P, Barasch J 2005 Endocytic delivery of lipocalin-siderophore-iron complex rescues the kidney from ischemia-reperfusion injury. J Clin Invest 115:610-621

16. Fjaertoft G, Foucard T, Xu S, Venge P 2005 Human neutrophil lipocalin (HNL) as a diagnostic tool in children with acute infections: a study of the kinetic. Acta Paediatr 94:661-666

17. Zappitelli M, Washburn KK, Arikan AA, Loftis L, Ma Q, Devarajan P, Parikh CR, Goldstein SL 2007 Urine neutrophil gelatinase-associated lipocalin as an early marker of acute kidney injury in critically ill children: a prospective cohort study. Crit Care 11:R84

18. Flo TH, Smith KD, Sato S, Rodriguez DJ, Holmes MA, Strong RK, Akira S, Aderem A 2004 Lipocalin 2 mediates an innate immune response to bacterial infection by sequestrating iron. Nature 432:917-921

19. Parravicini E, Lorenz JM, Nemerofsky SL, O'Rourke M, Barasch J, Bateman D 2009 Reference range of urinary neutrophil gelatinase-associated lipocalin in very low birth weight infants: preliminary data. Am J Perinatol 26:437-440

20. Huynh TK, Bateman DA, Parravicini E, Lorenz JM, Nemerofsky SL, Sise ME, Bowman TM, Polesana E, Barasch JM 2009 Reference values of urinary neutrophil gelatinase-associated lipocalin in very low birth weight infants. Pediatr Res 66:528532

21. Yan L, Borregaard N, Kjeldsen L, Moses MA 2001 The high molecular weight urinary matrix metalloproteinase (MMP) activity is a complex of gelatinase B/MMP-9 and neutrophil gelatinase-associated lipocalin (NGAL). Modulation of MMP-9 activity by NGAL. J Biol Chem 276:37258-37265

22. Cowland JB, Sørensen OE, Sehested M, Borregaard N 2003 Neutrophil gelatinaseassociated lipocalin is up-regulated in human epithelial cells by IL-1 beta, but not by TNF-alpha. J Immunol 171:6630-6639

23. Nickolas TL, O'Rourke MJ, Yang J, Sise ME, Canetta PA, Barasch N, Buchen C, Khan F, Mori K, Giglio J, Devarajan P, Barasch JM 2008 Sensitivity and specificity of a single emergency department measurement of urinary neutrophil gelatinaseassociated lipocalin for diagnosing acute kidney injury. Ann Intern Med 148:810819

24. Akcan-Arikan A, Zappitelli M, Loftis LL, Washburn KK, Jefferson LS, Goldstein SL 2007 Modified RIFLE criteria in critically ill children with acute kidney injury. Kidney Int 71:1028-1035 